\title{
Pulmonary embolism in patients with COVID-19 pneumonia on adequate oral anticoagulation
}

\author{
Giuseppe Di Tano ${ }^{1} \cdot$ Meghi Dede $^{2} \cdot$ Irene Pellicelli $^{3}$ - Enrico Martinelli ${ }^{3} \cdot$ Luigi Moschini $^{1} \cdot$ Elva Calvaruso $^{1}$. \\ Gian Battista Danzi ${ }^{1,4}(0)$
}

Accepted: 13 October 2021 / Published online: 27 October 2021

(c) The Author(s), under exclusive licence to Springer Science+Business Media, LLC, part of Springer Nature 2021

\begin{abstract}
Thrombotic complications are common in patients with severe COVID-19 pneumonia with important consequences on the diagnostic and therapeutic management. We report a consecutive series of five patients on long-term oral anticoagulation therapy who presented to our hospital for severe COVID-19 pneumonia associated with segmental acute pulmonary embolism despite adherence to therapy and with an adequate anticoagulant range at the time of the event. Four patients were receiving a direct oral anticoagulant (two with edoxaban, one with rivaroxaban and one with apixaban) and one patient a vitamin $\mathrm{K}$ antagonist. No significant thrombotic risk factors, active cancer, or detectable venous thromboembolism were present. In all cases, elevated d-dimer and fibrinogen levels with a parallel rise in markers of inflammation were documented. The combination of these findings seems to support the hypothesis that considers the local vascular damage determined by severe viral infection as the main trigger of thrombi detected in the lungs, rather than emboli from peripheral veins.
\end{abstract}

Keywords Pulmonary embolism $\cdot$ Infectious diseases $\cdot$ Anticoagulant

\section{Highlights}

- COVID-19 infection is associated with frequent thromboembolic events.

- Systemic anticoagulant therapy appears to be generally useful.

- Despite appropriate chronic anticoagulant therapy, segmental and subsegmental acute pulmonary embolism is documented.

- In this specific clinical scenario, the embolic genesis of pulmonary embolism is uncertain and complex interac-

Gian Battista Danzi

gbdanzi@gmail.com

Cardiology Unit, Cremona Hospital, Cremona, Italy

2 Maxillo-Facial Surgery Unit, Head and Neck Department, Santi Paolo e Carlo University Hospital, Università degli Studi di Milano, Milan, Italy

3 Pneumology Unit, Cremona Hospital, Cremona, Italy

4 Divisione di Cardiologia, Ospedale di Cremona, Viale Concordia, 1, 26012 Cremona, Italy tions between inflammation and coagulation play a major role.

\section{Introduction}

The COVID-19 does not involve only the respiratory tract, but has also major implications for the cardiovascular system [1]. Recently, accumulating data have shown an increased number of thromboembolic events among hospitalized patients which seems to suggest that coagulation function may be significantly impaired $[2,3]$ with important consequences on the diagnostic and therapeutic management of these patients [4]. Some studies pointed out that a dysregulated immune response, as seen during the inflammatory storm associated with COVID-19, could play a decisive role in endothelial dysfunction and thrombosis [5, 6], and microvascular permeability is crucial in viral infections. This condition is aggravated by hypoxia, which augments thrombosis by both increasing blood viscosity and hypoxia-inducible transcription factor-dependent signaling pathway [7].

Recently, clinical and autoptic studies reported a high rate of thrombotic complications in hospitalized patients, despite 
systematic prophylactic [8] or therapeutic anticoagulation $[9,10]$.

We report a consecutive series of five patients on longterm oral anticoagulation therapy who presented to our hospital for severe COVID-19 pneumonia associated with segmental pulmonary embolism (PE) despite a correct adherence to therapy and with adequate anticoagulant range at the time of the event. Our observations seem to reinforce the hypothesis of inflammatory-procoagulant pathogenesis undermining the clinical manifestations of COVID-19 which appear to be not fully protected by conventional anticoagulant treatment.

\section{Case reports}

We analyzed demographic, clinical, radiological and laboratory characteristics of five consecutive patients on chronic anticoagulation therapy who presented in the emergency department (between March 1st and May 5th 2020) for severe COVID-19 pneumonia. Pneumonia was associated with acute PE diagnosed by means of CT pulmonary angiography (CTPA) at the time of admission (Fig. 1). According to our Institutional protocol, CTPA was performed in all subjects who met a Geneva score $>10$ [11], and/or when the clinical suspicion of PE was very high, based on inadequate response to ventilation, or to high-flow oxygen therapy.
CTPA was performed by a 64-row multidetector computed tomography scanner. The scan included the entire chest, with a cranio-caudal direction of scanning, and supine patient position. Standard CT scan settings were adopted: $120 \mathrm{kV}$ tube voltage; $100-300 \mathrm{~mA}$ for tube current; $0.5 \mathrm{~s}$ of gantry rotation time; $64 \times 0.6 \mathrm{~mm}$ detector collimation. Vessel attenuation was obtained by injecting $50-70 \mathrm{~mL}$ (volume calculated based on patient's body weight) of non-ionic iodinated contrast media (iopamidol, Iopamiron $370 \mathrm{mg}$ iodine/ $\mathrm{mL}$; Bracco, Milan, Italy) followed by a saline chaser of $25 \mathrm{~mL}$ through an antecubital vein at a flow rate of 3-4 $\mathrm{mL} / \mathrm{s}$, using a mechanical power injector. CT acquisition was timed by using bolus-tracking technique with a regionof-interest (ROI) placement in the pulmonary trunk. Once a threshold of 100 Hounsfield Units (HU) was reached, the scan automatically started after a delay of $5 \mathrm{~s}$. Images were reconstructed with a thickness and increment of $1.25 \mathrm{~mm}$. All CTPA scans were interpreted by two experienced radiologists and disagreements were resolved through discussion until consensus was reached.

SARS-CoV2 infection was confirmed by positive nasopharyngeal swab on real-time reverse transcriptase-polymerase chain reaction assay. Four of them were on chronic direct oral anticoagulants (DOAC) therapy (two patients were receiving edoxaban, one patient rivaroxaban, and one patient apixaban) and one was receiving a vitamin $\mathrm{K}$ antagonist (VKA). At admission, all patients claimed to diligently
Fig. 1 Panel A and B Chest CT scan showing diffuse areas of consolidations and interlobular and intralobular septal thickenings involving both lung parenchymas. Panel $\mathbf{C}$ and $\mathbf{D}$ CT pulmonary angiography in axial view showing filling defects involving the inferior branch of the right pulmonary artery (open circles) and the posterior basal segmental branch of the left pulmonary artery (open circle)
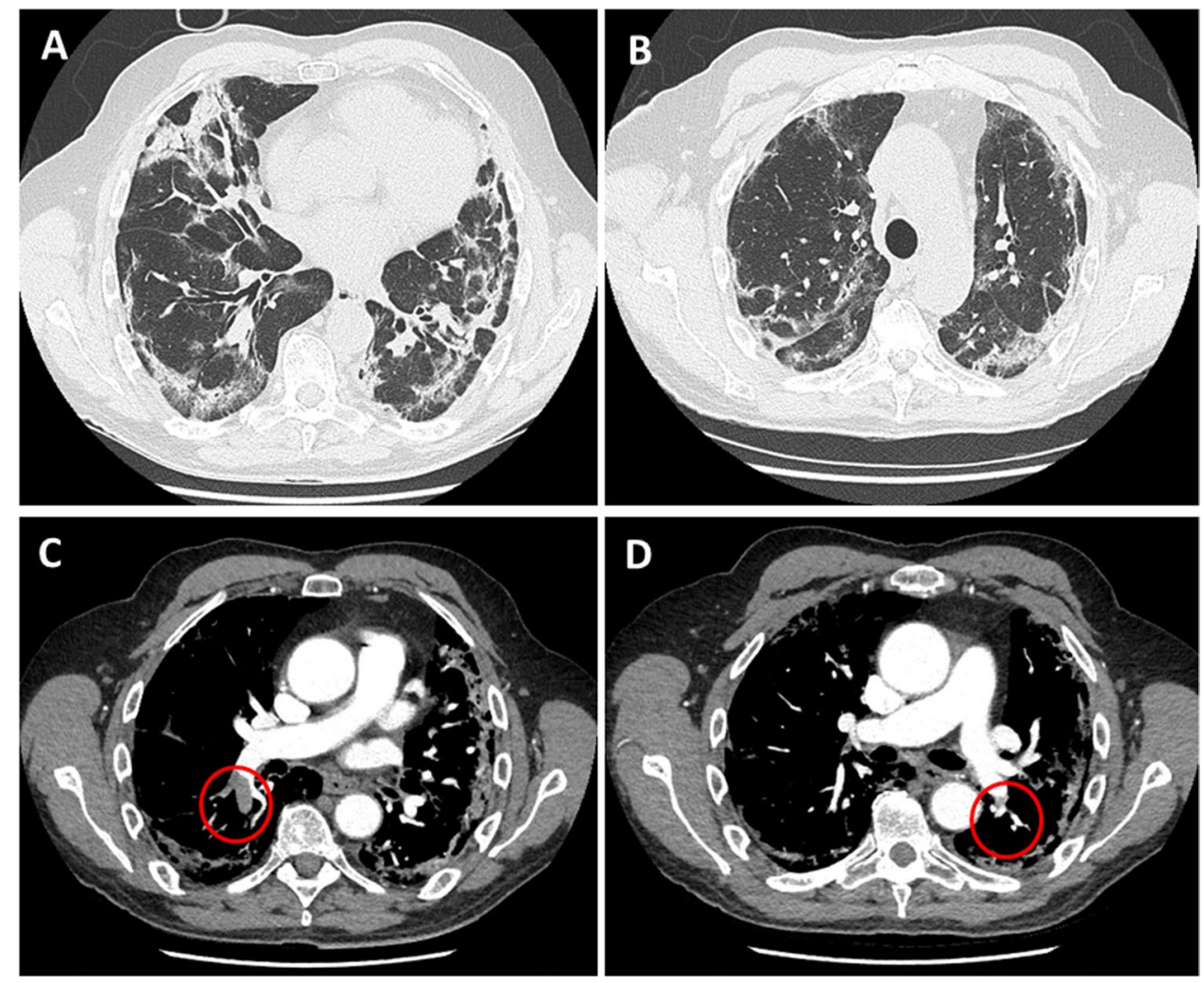
take the drugs, and at the same, time plasma samples were collected (an average of $15 \mathrm{~h}$ after the last dose intake). Appropriated DOAC plasma levels, and a prothrombin time, international normalized ratio (PT INR) $>2$, with a time in therapeutic range (TTR) $>75 \%$ were confirmed by laboratory findings in all cases (Table 1). DOAC levels measurements (expressed as drug concentration-equivalent in $\mathrm{ng} / \mathrm{ml}$ ) were evaluated by using a specific anti FXa assay calibrated for apixaban, edoxaban, and rivaroxaban (Atellica COAG 360TM, Stago, France) [12]. At the time of admission, oral anticoagulant therapy was promptly replaced with a full dose (100UI/kg bid) of subcutaneous low-molecular-weight heparin up to one month of follow-up.

The chronic anticoagulant treatment had been previously prescribed as primary prevention of atrial fibrillation (AF)-related stroke in three patients and as secondary prevention of venous thromboembolism (VTE) in the remaining two patients. The dosages of DOACS were prescribed for each drug according to age, weight, and renal function as recommended by the international guidelines [13]. Two patients received anticoagulation after an episode of PE that was a consequence of a deep venous thrombosis (DVT) as a complication of a fracture of the lower limb. These episodes have occurred one year and six months before the present hospitalization. No other predisposing or genetic factors were reported. Demographic, comorbidities data, and main risk factors were recorded, as well as the main clinical features (ARDS severity by $\mathrm{PaO} 2 / \mathrm{FIO} 2$ ratio, according to Berlin definition [14], PE type based on CTPA description, laboratory findings (including coagulation markers) and clinical outcomes. All the collected data are summarized in Table 1. All patients underwent lower extremities doppler ultrasonography but no sign of deep vein thrombosis (DVT) was detected. Transthoracic echocardiography showed no dilatation of right ventricular or pulmonary hypertension.

Table 1 Demographic, clinical, radiological and laboratory findings

\begin{tabular}{|c|c|c|c|c|c|}
\hline & Case 1 & Case 2 & Case 3 & Case 4 & Case 5 \\
\hline Age (years) & 86 & 67 & 63 & 83 & 86 \\
\hline Gender & M & M & M & $\mathrm{F}$ & $\mathrm{F}$ \\
\hline Weight (Kg) & 68 & 65 & 72 & 49 & 60 \\
\hline BMI > 30 & - & - & - & - & - \\
\hline Hypertension & + & - & - & + & - \\
\hline Diabetes & + & - & - & - & + \\
\hline Heart failure & + & - & - & + & - \\
\hline COPD & + & + & - & - & + \\
\hline Active cancer & - & - & - & - & - \\
\hline Chronic renal failure & + & - & - & - & - \\
\hline Anticoagulant drug & Rivaroxaban & Edoxaban & Apixaban & Edoxaban & Warfarin \\
\hline Anticoagulant dose & $15 \mathrm{mg}$ day & $60 \mathrm{mg}$ day & $5 \mathrm{mg}$ BID & $30 \mathrm{mg}$ day & \\
\hline Plasma drug concentration $\mathrm{ng} / \mathrm{ml}$ & 68 (LloD: 25) & 54 (LloD: 20) & 48 (LloD: 25) & 62 (LloD: 20) & \\
\hline Indication to anticoagulation & $\mathrm{AF}$ & Previous PE & Previous PE & $\mathrm{AF}$ & $\mathrm{AF}$ \\
\hline PE distribution & Segmental & Segmental & Subsegmental & Subsegmental & Subsegmental \\
\hline ARDS severity at admission & Mild & Severe & Mild & Severe & Mild \\
\hline $\mathrm{PaO} 2 / \mathrm{FIO} 2$ ratio & 187 & 93 & 178 & 88 & 190 \\
\hline PLT count $\times 10^{3}$ & 283 & 116 & 160 & 269 & 215 \\
\hline CRP mg/l (nv: <5) & 70.5 & 82 & 33.2 & 321 & 27.3 \\
\hline D-dimer $\mu \mathrm{g} / \mathrm{ml}(\mathrm{nv}: 0.5$ ) & 3.99 & 18.4 & 7.2 & 0.27 & 1.7 \\
\hline LDH U/l (nv: <248) & 234 & 786 & 214 & 447 & 358 \\
\hline INR & 1.85 & 1.03 & 1.18 & 2.69 & 2.33 \\
\hline APTT (nv: < 1.2) & 1.37 & 0.99 & 1.01 & 1.59 & 1.62 \\
\hline $\operatorname{TTr}(\mathrm{nv}:<1.2)$ & 1.10 & 1.3 & 1.60 & 1.2 & 0.9 \\
\hline Fibrinogen ml/dl (nv: < 200) & 348 & 476 & 365 & 286 & 210 \\
\hline Creatinine mg/dl (nv: < 1.18) & 1.28 & 1.09 & 0.86 & 0.74 & 0.6 \\
\hline eGFR (mL/min) & 39.8 & 60.5 & 89 & 44.6 & 63.8 \\
\hline
\end{tabular}

$B M I$ body mass index, $A F$ atrial fibrillation, $P E$ pulmonary embolism, $A R D S$ acute respiratory distress syndrome, $P a O 2$ arterial partial pressure of oxygen, FIO2 fraction of inspired oxygen, PLT platelet, CRP C-reactive protein, $L D H$ lactate dehydrogenase, INR international normalized ratio, APTT activated partial thromboplastin time, $T T r$ thrombin time ratio, $L l o D$ lower limit of detection, $e G F R$ estimated glomerular filtration rate 


\section{Discussion}

Increased thromboembolic events among COVID-19 hospitalized patients have been largely reported [2, 3]. A French experience observed a frequency of PE twice higher than that found in a control period [15]. Additionally, clinical [16] and autopsy studies of patients who died of COVID-19 described a high incidence of deep venous thrombosis, with one-third of the patients who had a pulmonary embolism as the direct cause of death [17].

Approximately $1-2 \%$ of the western population is chronically treated with an oral anticoagulant [18], but, in the pre-COVID-19 era thrombotic events in patients on anticoagulation therapy were pretty uncommon [19]. In the PREFER Registry, among patients receiving chronic anticoagulation for AF (VKA in $48.7 \%$ of cases and a DOAC in $24.5 \%$ ), the DVT recurrence was $3.9 \%$ with an overall incidence of PE of $1.3 \%$ [20]. Furthermore, only one recurrent episode of VTE (1.7 events per 100 patientyears) was documented in the more recent Dresden NOAC Registry [21]. However, COVID-19 infection is now considered an additional risk factor for DVT in hospitalized patients, but the rate of thrombotic recurrence in treated patients is unknown.

We report a series of five consecutive patients on chronic anticoagulant therapy who presented to our emergency department with severe COVID-19 pneumonia associated with acute PE. The peculiarity of this population is characterized by absence of major predisposing thrombotic risk factors: lack of detectable or recurrent history of VTE; no signs of active cancer or impairment of renal function; elevated D-dimer and fibrinogen levels with a parallel rise in markers of inflammation; localization of thrombus in the segmental and subsegmental branches of pulmonary arteries. Even if our observation is concerning a small sample of patients, these findings seem to support the hypothesis of a local vascular damage determined by viral infection in severe COVID-19 as the main trigger of thrombi detected in the lungs, rather than emboli from peripheral veins [22]. Recent morphologic studies may corroborate this pathological mechanism. In a series of autopsies of deceased COVID-19 patients [10, 23], pulmonary arteries at the hilum were free of thrombo-emboli, moreover, an elevated rate of platelet-fibrin thrombi in small arterial vessels $(<$ $1 \mathrm{~mm}$ ) was documented in the lung periphery, along with inflammatory infiltrate composed by macrophages in alveolar lumens and lymphocytes. This etiopathogenetic mechanism based on intense inflammatory-hypercoagulative status could explain the lack of protective effects of oral anticoagulant drugs (DOACS and VKA) despite their correct dose of administration and the evidence of adequate INR values and plasma levels. The latter were obtained by means of a validated specific anti FXa assay [12] and not via mass-spectrometry [24]. Consequently, we should cautiously consider the findings of DOACS concentration in this limited sample size.

In conclusion, recent observations suggest that COVID19 infection promotes hypercoagulability. Our case series of severe interstitial pneumonia associated with acute PE in patients chronically treated with oral anticoagulant therapy underlines the role of complex interactions between inflammation and coagulation, representing a further challenge in the treatment of COVID-19 disease.

Author contributions GDT designed the study; MD and IP contributed to data collection; all authors contributed to the interpretation of the data collected; GDT, MD and GBT wrote the draft of the manuscript; all the authors contributed to the final revision and accepted the manuscript.

\section{Declarations}

Conflict of interest None of the authors has a conflict of interest to declare.

\section{References}

1. Guo T, Fan Y, Chen M et al (2020) Cardiovascular implications of fatal outcomes of patients with coronarovirus disease 2019 (COVID-19). JAMA Cardiol 5:811-818

2. Bikdeli B, Madhavan MV, Jimenez D (2020) e al. COVID-19 and Thrombotic or Thromboembolic Disease: Implications for prevention, antithrombotic therapy, and follow-up. J Am Coll Cardiol 75:2950-2973

3. Danzi GB, Loffi M, Galeazzi G, Gherbesi E (2020) Acute pulmonary embolism and COVID-19 pneumonia: a random association? Eur Heart J 41:1858

4. Tang N, Bai H, Chen X et al (2020) Anticoagulant treatment is associated with decreased mortality in severe coronavirus disease 2019 patients with coagulopathy. J Thromb Haemost 18:1094-1099

5. Schulz C, Engelmann B, Massberg S (2013) Crossroads of coagulation and innate immunity: the case of deep vein thrombosis. J Thromb Haemost 11(Suppl 1):233-241

6. Mezger M, Nording H, Sauter R et al (2019) Platelets and Immune Responses During Thromboinflammation. Front Immunol 10:1731

7. Varga Z, Flammer AJ, Steiger P et al (2020) Endothelial cell infection and endotheliitis in COVID-19. Lancet 395:1417-1418

8. Klok FA, Kruip MJHA, van der Meer NJM et al (2020) Incidence of thrombotic complications in critically ill ICU patients with COVID-19. Thromb Res 191:145-147

9. Llitjos JF, Leclerc M, Chochois C et al (2020) High incidence of venous thromboembolic events in anticoagulated severe COVID19 patients. Thromb Haemost 18:1743-1746

10. Lax SF, Skok K, Zechner P et al (2020) Pulmonary arterial thrombosis in COVID-19 with fatal outcome:results from a prospectiv, single-center, clinicopatholoogic case series. Ann Intern Med 173:350-361 
11. Klok FA, Mos ICM, Nijkeuter M et al (2008) Simplification of the revised Geneva score for assessing clinical probability of pulmonary embolism. Arch Intern Med 168:2131-2136

12. Gosselin RC, Adcock DM, Bates SM et al (2018) International council for standardization in Hematology (ICSH). Recommendations for laboratory measurement of direct oral anticoagulants. $\mathrm{J}$ Thromb Haemost 118:437-450

13. Steffel TJ, Verhamme P, Tatjana S. Potpara TS et al (2018) The 2018 European Heart Rhythm Association Practical Guide on the use of non-vitamin $\mathrm{K}$ antagonist oral anticoagulants in patients with atrial fibrillation. Eur Heart J 39:1330-1393

14. Ranieri VM, Rubenfeld GD, Thompson BT et al (2012) Acute respiratory distress syndrome. JAMA 307:2526-2533

15. Poissy J, Goutay J, Caplan M et al (2020) Pulmonary embolism in COVID-19 patients: awareness of an invcreased prevalence. Circulation 142:184-186

16. Zhang L, Fenf X, Zhang D et al (2020) Deep Vein Thrombosis in Hospitalized Patients with Coronavirus Disease 2019 (COVID19) in Wuhan, China: Prevalence, Risk Factors, and Outcome. Circulation 142:114-128

17. Fox SE, Akmatbekov A, Harbert JL et al (2020) Pulmonary and cardiac pathology in COVID-19: The first autopsy series from new orleans. Lancet Respir Med 8(7):681-686

18. Testa S, Paoletti O, Giorgi-Pierfranceschi M, Pan A (2020) Switch from oral anticoagulants to parenteral heparin in SARS-CoV-2 hospitalized patients. Intern Emerg Med 15:751-753
19. Schulman S (2017) How I treat recurrent venous thromboembolism in patients receiving anticoagulant therapy. Blood 129:3285-3293

20. Cohen AT, Gitt AK, Bauersachs R et al (2017) The management of acute venous thromboembolism in clinical practice. Results from the European PREFER in VTE Registry. J Thromb Haemost 117:1326-1337

21. Beyer-Westendorf J, Förster K, Pannach S et al (2014) Management, and outcome of rivaroxaban bleeding in daily care: results from the Dresden NOAC Registry. Blood 124:955-962

22. Cattaneo M, Bertinato EM, Birocchi S, Brizio et al (2020) Pulmonary embolism or pulmonary thrombosis in COVID-19? Is the recommendation to ude high-dose heparin for thromboprophylaxis justified. J Thromb Haemost 120:1230-1232

23. Wichmann D, Sperhake JP, Lütgehetmann M et al (2020) Autopsy Findings and Venous Thromboembolism in Patients With COVID19: A Prospective Cohort Study. Ann Intern Med. https://doi.org/ 10.7326/M20-2003

24. Douxfils J, Gosselin RC (2017) Laboratory Assessment of Direct Oral Anticoagulants. Semin Thromb Hemost 43:277-290

Publisher's Note Springer Nature remains neutral with regard to jurisdictional claims in published maps and institutional affiliations. 